Ist lumbar vertebræ. A bone-grafting operation was done, and she was cured of her pain. In recent cases good results may be obtained by hyperextension of the spine and the application of a plaster jacket, the displacement being actually reduced by suspending the patient with the spine hyperextended. In late and chronic cases a spinal fusion operation should be done at once.

\title{
Conclusion.
}

Diseases of the vertebral column in general do not come within the scope of this lecture. Tuberculosis, syphilis, and new growths of the vertebræ are distinguished from the foregoing conditions by the severity of their symptoms and the early clinical and radiographical evidence of gross bone destruction. The conditions dealt with are for the most part simple traumatic lesions which are easily recognized by a few characteristic signs and which usually respond readily to appropriate treatment. To these I have added osteoarthritis of the lumbar spine which is often traumatic in origin or may be associated with a more generalized senile osteoarthritis. These conditions together are responsible for the great majority of cases of persistent pain in the lower part of the back or "low back pain."

\section{THE EFFECTS ON THE HEART OF DISEASE OF OTHER SYSTEMS, AND INDICATIONS IN TREATMENT.}

\author{
BY A. J. SCOTT PINCHIN, M.D., F.R.C.P. \\ [Abridged.]
}

A consideration of the reaction of the heart to systemic disease involves a brief recapitulation of the structures of the heart; in certain diseases the incidence may be mainly on one structure whilst in others all parts may tend to be affected. The fibrous supporting tissue of the heart, the valvular structures, the peri-arterial adventitia (which is especially involved in rheumatic disease), the muscular tissue with its characteristics of tonicity, excitability, its capability of response by hypertrophy to extra work and its tendency to atrophy when the load is lifted; the nodes and bundle which may be involved specifically in certain diseases: syphilis, rheumatism, diphtheria and pneumonia. Lastly the nervous control of the heart by the vagus and sympathetic, examples of which occur in diphtheritic neuritis of the vagus, and sympathetic stimulation in thyrotoxicosis.

A résumé of the effects of disease such as I propose to put before you cannot be more than a somewhat superficial discussion of the principal features. Nevertheless it is a subject $I$ do not remember having seen used as the matter of a lecture, which cannot be said of many subjects of recent years; and so it seemed it might be useful to review how disturbances by disease elsewhere may affect the heart.

The muscles and interstitial tissues of the heart seem in certain diseases to be particularly liable to attack.

Rheumatic fever is a disease so closely allied to heart disease that it is perhaps not fair to include it in such a summary. However, it is a general disease, and its effect on the fibrous structures, especially in the heart, where it manifests itself by the damage to valves and the formation in the supporting tissues (via the periadventitia of the smaller hlood-vessels) of Aschoff's nodes, is of so much importance that it must be stressed. 
The nodes, similar to the rheumatic nodule found elsewhere in active cases, are usually regarded as diagnostic of rheumatic disease when found in the cardiac musculature. $\frac{\mathrm{D}}{\mathrm{D}}$ These nodes, if near the conducting tissue, may press on the bundle and so cause an $\stackrel{.}{.}$ interference with conduction and complete or partial heart block. In two specimens of heart block at Victoria Park Hospital the endocardium is seen to be plentifully spotted with miliary nodules.

I have mentioned rheumatism in the first instance as it is the disease of all others $\frac{\frac{5}{\vec{p}}}{\vec{\sigma}}$ which seems to have its main incidence on the heart, as typhoid has on the intestine $\stackrel{\varnothing}{\circ}$ and the pneumococcus on the lung. In most other diseases the result is secondary to ${ }^{\infty}$ disease elsewhere, either of the toxins, endogenous or exogenus, or damage due to $\vec{\circ}$ organisms.

The toxin of tuberculosis has interesting effects on the heart. In the tuberculous patient the heart is nearly always small, vertical and central in position, and so commonly is this met with that it has been called the tuberculous type of heart. It is 6

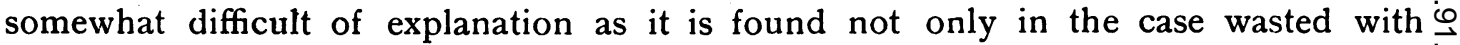
tubercle, but in the still fairly healthy individual, and it occurs even in those fibroid chronic tubercular cases in which one would imagine there might be some hypertrophy. In fact, it may be of use in the diagnosis of a fibrosis which has become infected with tuberculosis from a fibroid tubercle.

The toxin of this disease usually also causes a rise in the rate of the heart and a lowered blood-pressure, apart from any febrile reaction. Many cases of so-called $\vec{\oplus}$ D.A.H. during the War (which classification usually meant accelerated action of unexplained origin) proved after some years to have been a cryptic tubercular infectio and probably many were cryptic hyperthyroid. The disease seldom causes any cardia lesion from invasion of the micro-organism, except as direct spread from pulmonary or mediastinal infection, the pericardium being the part affected, either as a tuberculous pericarditis or occasionally as a part of a polyserositis. When an effusion forms the onset is insidious and the condition may be missed, and it is sometimes followed by severe mediastinal pericarditis.

Miliary tubercles may be found in the heart in miliary tuberculosis, but are of noimport in view of the prognosis of the systemic disease, whilst anything in the nature of a tuberculoma is of extreme rarity.

There is a point of great importance in connection with the heart and pulmonary tubercle, and this is the fact that pulmonary tuberculosis is almost unheard of in mitral stenosis. Probably the passive congestion of the lungs caused by this disease produces 9 early cure if infection does take place.

One cannot leave the subject of tuberculosis without mentioning the effect on the heart of artificial pneumothorax treatment. A unilateral artificial pneumothorax appears. to have little effect on the heart, but if kept up too long the consequential fibrosis may $\tilde{V}^{\circ}$ be so extreme as to cause displacement of the heart of such an extensive degree that it $\omega$ incommodes its action. This displacement disability is also seen in scoliosis. In double artificial pneumothorax, if kept up for too long a right-sided failure of the heart is produced from which the patient dies.

Chronic malarial infections seem to produce a degenerative condition of the heart- $\frac{0}{0}$ muscle and softening of the interstitial tissue, producing one type of the large flabby mushroom heart, without a definite myocarditis. 
On the other hand, certain bacterial infections produce a well-marked myocarditis. This is particularly noticeable in bacillary dysentery due to the Shiga organisms; as in some other types, the right side of the heart seems to feel the effect more than the left, possibly owing to the lesser mass of muscle and the fact that during the disease whilst the left heart has less to do the right is not relieved of any load.

Several times in this disease I have seen patients die of an acute right-sided failure. At the post mortem in these cases the right side of the heart was distended in a globular fashion whilst the lungs were completely empty of blood and crepitant. The effect on the left side of the heart is evident when the patient begins to get about-it takes a long while to recover the exercise tolerance.

In connection with amœbic dysentery it should be noted that emetine has a poisonous effect on the myocardium and it is not advisable to allow the patient to go about whilst he is having treatment with this drug. The blood-pressure sinks whilst the drug is being given.

One of the most important diseases in view of its action upon the heart is diphtheria, and though this has been rendered much less serious by the use of antitoxin it may still have a disastrous effect in children in whom the disease is not recognized, or in cryptic types of the disease. The effect on the heart is caused by the toxin which produces a degenerative myocarditis and sometimes a vagal neuritis. Though heart block has been recorded it.is a very rare complication. In an affected heart the danger is real and the case must be treated with absolute rest, intravenous glucose solution, and in view of the fact that the toxins of diphtheria are particularly poisonous to the adrenals (a fact which seems also to apply to the toxin of bacillary dysentery), adrenalin should be used.

The symptoms are those of the feeble cardiac action, pallor or sometimes cyanosis, enlargement of the heart and tachycardia or bradycardia, the latter usually being a wellmarked sign in convalescence. The condition may remain serious for weeks and any shock or exertion produce sudden death.

In contradistinction to this disease is enteric fever, which, although rarely the the cause of a bacterial endocarditis, usually only causes wasting with slight degenerative changes in the heart-muscle, the condition produced being one of extreme shock and asthenia together with the usual low blood-pressure.

Though it is denied by some that the pneumococcus has any marked effect on the heart-muscle; though the organism may rarely cause an endocarditis ; yet there must be few who feel happy as regards the cardiac condition in bad cases of pneumonia due to this organism, and this before the crisis (which indeed is not a frequent occurrence in the present day), at which stage the vasomotor control gives out and causes a loss of cardiac tonus in consequence.

The right side of the heart may be badly overloaded mechanically owing to the pulmonary circuit being partially closed ; this may be temporarily relieved by venesection.

The work of Dixon on the effect of digitalis in pneumococcal infections would seem to show that the exhibition of fresh tincture or infusion of digitalis early in the disease prevents the toxin from acting on the heart-muscle, and goes to show that the empiricism of some of us who have always believed in digitalis in the early stages of the disease is not without a solid basis. But it must be realized that this drug is useless, or possibly dangerous, when the myocardium has become intoxicated. Heart block is very rare as a complication of pneumonia, and as the principal action of digitalis is on the bundle 
there seems no contra-indication to its use in this way as a guard to the cardiac muscle against intoxication.

In the perhaps more common streptococcal pneumonia and influenzal infections sudden death is not uncommon, and one would suspect the conclusion made by some that the cause is in the nature of an effort syndrome due to the severe systemic disturbance and shock of disease rather than the effect on the heart-muscle itself. Cardiograms taken of influenzal cases sometimes show evidence of extensive myocardial lesions, presenting a picture such as is occasionally seen in quinidine poisoning.

A point worthy of note and difficult of explanation is the effect of various infections, in the invasion stage, in causing a slowing of the pulse, and the best-known example of this is in the enteric group.

In many other diseases, bacillary dysentery, Malta and sandfly fever, influenzal infections, and so forth, the period of invasion may be accompanied by a slow pulse ; yet, although often in streptococcal infections the pulse is slow, in scarlet fever a rise of pulse-rate out of proportion to the temperature is a useful diagnostic feature.

An autogenous toxic slowing is, of course, met with in jaundice.

Disease and conditions of life may affect the heart indirectly through their action on the endocrine glands.

Toxins of disease may destroy the secreting powers and the structure itself of the adrenals, whilst tuberculosis and new growth, commonly secondary to thoracic neoplasm, may destroy the gland.

In addition to this, stress of life may lead to over-activity of the adrenal.

Adrenalin is necessary for the activity of the sympathetic system, it is a powerfue stimulant to the heart mainly by constricting peripheral vessels and increasing tonus, whilst if injected into the circulation of a patient under influence of chloroform, it may cause ventricular fibrillation. If the adrenal secretion is absent there is marked hypotension and hypotonus of heart-muscle which is affected as the other muscles in this disease-the whole circulation is in a continued condition of shock.

Continued stimulation of the adrenal as in constant apprehension, a condition seen frequently during the War, is accompanied by hypertension and a disturbance generally of the sympathetic shown most markedly in the thyroid, over-action of which gland was often seen in women during these years.

Disturbances of the thyroid function is indeed a very frequent cause of cardiac disorders; many cases of sinus tachycardia are cases of Graves' disease forme fruste, where the other usually found symptoms may be absent, except tremor, which is nearly always present.

In more marked disturbance and hypersecretion, a condition of thyrotoxic heart is produced.

The increased cardiac action caused by the abnormal secretion is not solely due to more active metabolism, the toxin appearing to have a specific action on the heart, and not infrequently in long-standing cases the rate of the heart and its general disturbance remains fixed after the cause, i.e., the thyroid, has been removed. In old people who have had thyroid disease which has become quiescent the after-effects of this poison may be found. In the early stages the blood-pressure is slightly increased, the diastolic normal, and therefore there is a slight increase of the pulse-pressure. Later, 
the pressure may fall, to rise often to a high figure, when the heart begins to fail and fibrillation appears. The heart is often apparently enlarged on account of force of beat, but hypertrophy, apart from the later cardiac derangements, is uncommon.

Paroxysmal sino-auricular tachycardia, auricular tachycardia and auricular fibrillation may occur. The first and last are the most common, and auricular fibrillation when the heart begins to tail is met with frequently. When the thyroid disease is of long-standing, attempts to restore normal rhythm by quinidine is useless, unless operation is proposed, as the causative condition remains.

Goodall states that in the cardiogram large $P$. and $T$. with $T .=$ to $P$. is suggestive of thyrotoxicosis, but this is not universally acknowledged. (Though the P. and T. and whole complex are of low voltage in myxœdema.)

There seems no doubt that the treatment of all cases, except those mild ones which respond to rest, is operation before secondary cardiac changes render the prognosis of operation materially worse, or the toxin permanently poisons the heart.

Iodine is best used in preparation for operation, though many mild cases respond well to continued iodine treatment. $\mathrm{X}$-ray is not very satisfactory as a permanent measure, and in toxic adenoma may cause an exacerbation of symptoms of a severe degree.

Focal infections, by causing a septicæmia, may give rise to a bacterial endocarditis. It is probable that the valves must have been previously damaged for this to take place, and it is unlikely to occur as a sequel apart from a septicæmia. The infections that may lead to this complication are many: tonsils, apical tooth abscesses and infected tooth sockets, middle-ear disease, cholecystitis, prostatitis, gonorrhœa, \&c.

Apart from this, focal sepsis, particularly if under tension as in sinuses, teeth abscesses and ch. fibrosed tonsils may especially cause cardiac symptoms, tachycardia, extra-systoles and cardiac pain. Sepsis in the old may cause fibrillation; this is a frequent difficulty in old patients with prostatic disease, cystitis and renal disability.

These conditions from every point of view should be attended to. Vaccines should not be given to a patient with pus in a closed cavity, and tooth extraction should not be done in the mass, a few being taken at a time. Septicæmia has been caused by the wholesale extraction of septic teeth. Probably one of the most potent causes of focal sepsis is found in the tonsil, particularly the old fibrotic type, which has been the seat of abscess and often has been guillotined in the past.

I have spoken of myocarditis in association with infectious diseases and other conditions. The term is somewhat loosely used, and should really be applied to those conditions in which there is an inflammatory reaction in the muscle or interstitial tissues. In the acute form the terminology is probably more accurate than in the chronic form in which various types of degenerative change, such as coronary sequelæ and hypertensive disease, tend to be included, though it might well be argued that these conditions cause an inflammatory reaction followed by fibrosis in the same way as an acute infection may.

It is difficult to draw a sharp line of demarcation between the acute and the chronic form, the one merges into the other, and the chronic may have periods when there is a light up of the inflammatory condition. - The whole subject seems to be rather vague in the minds of most authors, and there is a good deal of variation in opinion and stress laid on the subject. It has been suggested that the changes may be 
divided into parenchymatous, fatty, hyaline and amyloid, calcareous, fragmentation and interstitial.

In parenchymatous degeneration the muscle-fibres swell and lose their striation, the intermuscular fibrous tissue swells, but the muscle retains its contractile power in a lesser degree, and there may be a tendency to rupture. Such cases may be the result of of poison, such as phosphorus, or fatty degeneration, and in the degenerated tissues calcium salts are sometimes found. The changes are generally acknowledged to be earliest in the papillary muscles and the auricular ventricular ring, leading to functional deficiency of the valves. In addition to these changes there may be a periarteritis or endarteritis of the vessels, and, as mentioned above, perhaps the involvement of the interstitial tissue either as a softening or true inflammatory change.

A heart, the myocardium of which is affected by some inflammatory change, has a loss of tonicity and dilates easily to any load above the minimum which is put upon it, though it may be able to carry on efficiently whilst the patient is on complete rest; it being remembered that this means mental as well as physical rest.

The normal heart tends to become smaller on exercise, and it is denied by most that the normal heart will develop an acute dilatation with abnormal effort, though there is difficulty in proving that a heart is normal or that it is not abnormal if it does dilate in response to effort.

The dilatation in the diseased heart may be enormous, and the capacity of the heart to carry on its functions when extensively diseased is often surprising. I have myself seen a heart in which most of the muscle appeared to be replaced by malignant growth, although the patient had been getting about till the last.

The diagnosis of the acute form is usually not difficult in association with some disease in which it can be expected, though in a very acute toxic condition with pain it is sometimes difficult to be sure whether the condition may not be coronary disease.

The condition is usually accompanied by a falling blood-pressure due in part to the failing efficiency of the heart-muscle, but often associated with a deficiency of adrenal secretion. It must be remembered that in some diseases, especially diphtheria and Shiga dysentery, the adrenals are reduced to an empty shell. The blood-pressure may fall as low as 60 or 70 systolic. The heart may be either rapid or slow, but the characteristic of most value from the diagnostic point of view is the alteration in the sounds. These are short and clear, equal in intensity and equal in spacing, producing the fotal sounds or embryocardia ; occasionally a presystolic gallop rhythm is present. Differential stethoscopes of various types have been designed to measure the intensity of the two sounds, but in the acute case, at all events, are quite unnecessary.

If dilatation had taken place pulmonary, mitral and tricuspid murmurs are to be expected with an accentuation of the pulmoric second sound, and in addition attacks of severe precordial pain may occur.

Bradycardia is met with especially after diphtheria and pneumonia ; this may be primarily myocardial, or can be due to a vagal stimulation or to the effect of the toxins on the bundle producing a complete or partial heart block; Vincent having showed that diphtheria caused a neuritis of the cardiac plexus.

A large number of figures have been collected from diphtheria cases with very few showing heart block, which is much more commonly a sequel in rheumatic disease. It would appear that most bacterial diseases and many poisons affect the heart-muscle; 
amongst these are typhoid, rheumatism, diphtheria, scarlet fever, tubercle, septicæmia, dysentery, puerperal fever, all streptococcal infections, so-called influenza (not Pfeiffer's bacillus), alcohol and carbon monoxide poisoning.

As mentioned before, alteration in the heart sounds and collapse in the course of an infectious illness are the main points in diagnosis. Pericardial effusion must not be missed on this account.

Suspected cases must be examined frequently. An undulatory apex beat is not constant but usual if dilatation has occurred. Irregularities are common, either ectopic beats or dropped beats, and an associated tachycardia is a bad prognostic sign.

Death may be instantaneous following a syncopal attack, and this may occur either on the first occasion or on a subsequent one. As has already been mentioned right-sided failure occurs in dysentery and is present in a large proportion of myocardial deaths in pneumonia. Absolute rest is essential for prolonged periods until the pulse slows in tachycardia or accelerates in bradycardia, and the sounds become normal.

Digitalis in acute and chronic myocarditis is a dangerous drug and must only be given in small doses.

Strychnine increases the tonicity of the heart-muscle, stimulates the nerves, the respiratory and vasomotor functions.

Adrenalin increases the tonicity of the cardiovascular system and is particularly useful in those cases when there is reason to suspect the supply is short.

In my own opinion coramine and cardiazol are of value in those cases of low pressure after many diseases.

We have to recognize that there is little in the life of the individual which takes place which fails to have its repercussion on the heart ; hard manual work, especially lifting with closed glottis, hypertension from whatever cause; the anæmias and leukæmias which affect the heart, not only because greater rapidity of circulation must be kept up, but also because the diminished oxygen content affects the heart itself so that degenerative changes may occur from this cause. With the anæmias adventitious or functional murmurs are often heard so that it is quite possible to mistake a case of pernicious anæmia for aortic disease.

Plethora overloads the heart and causes hypertrophy as shown in the German beerdrinkers' heart; viscosity increase, as in polycythæmia vera, is another pathological condition. On the other hand diminution of fluid in the circulation, as in surgical shock or diseases such as cholera and dysentery, deprive the heart of the degree of tension which is necessary for a satisfactory contraction of the ventricle.

In the endeavour made to put before you these changes in the heart which are consequentive to systemic disease, one cannot help but realize that in most of these conditions it is a responsibility devolving on the general practitioner rather than the consultant. 